\title{
Study on Dynamic Response Characteristics of Rock Slope
}

\author{
FU Zhanling ${ }^{1}$, CAO Jingquan ${ }^{1}$, GAO Wuping ${ }^{1}$, YAO Xinqiang ${ }^{1}$ \\ ${ }^{1}$ Eathquake Administration of Tianjin, Tianjin, 300201
}

\begin{abstract}
After the wenchuan earthquake, the dynamic response law of rock slope under the action of dynamic load is a very concerned and urgent problem in the field of seismic engineering. This paper analyzed the dynamic response law of simplified rock slope under the cosine wave dynamic load by numerical simulation with FLAC $^{3 \mathrm{D}}$ software. This research emphatically analyzed the variation law of amplification coefficient of seismic displacement, acceleration and velocity of rock slope under the condition of different slope height, slope angle, dynamic load period and dynamic load amplitude, which provides a basis for reducing seismic damage of slope area and seismic design of engineering structure.
\end{abstract}

\section{Introduction}

China has a vast land area, and the mountainous areas account for about two-thirds of the total area of China, which objectively determines that there are a large number of slopes distributed in China. With the continuous development of China's economic construction, a large number of new artificial slopes appeared during the construction of "Western Development", "South-to-North Water Transfer Project", "Three Gorges Project" and "Four Vertical and Five Horizontal" Transportation Hubs. These slopes have different amplification effects on ground motion, which will directly threaten the safety of the projects themselves, and then cause harm to people's lives and properties in big cities and large enterprises.

Scholars at home and abroad have done some research on the response law of slope under earthquake. $\mathrm{Xu}$ Guangxing et al. ${ }^{[1]}$ studied the dynamic response law of soil slope model by using large shaking table simulation experiment. Xiao Ruihua et al. ${ }^{[2]}$ studied the dynamic response law of double-sided slope under strong earthquake by shaking table. Zhang Ping et al. ${ }^{[3]}$ studied the dynamic response characteristics of sand interlayer jointing through shaking table experiments. Takano Hideo $^{[4]}$ observed the seismic effect on the slope, and the results showed that the seismic intensity on the slope increased by about 1 degree relative to the valley bottom. In 1987, Wang Cunyu ${ }^{[5]}$ conducted a dynamic model test on Ertan Arch Dam to study the amplification effect of rock slope on earthquake. He Yunlong ${ }^{[6]}$ et al. (1998) studied the influence of rock slope height on earthquake amplification effect by dynamic finite element method. In 1991, the Changjiang River Scientific Research Institute used the finite element method to study the seismic stability of the high slope of the Three Gorges Ship Lock ${ }^{[7]}$. Li Yushu ${ }^{[8]}$ used the dynamic finite element method to study the full-time dynamic response law of the lateral slope at the unsymmetrically pressed tunnel entrance under horizontal earthquake, vertical earthquake and both horizontal and vertical earthquakes.

\section{Establishment of slope model and arrangement of monitoring points}

Choose simple slope models, respectively with slope heights of $30 \mathrm{~m}, 60 \mathrm{~m}, 150 \mathrm{~m}, 300 \mathrm{~m}$ and $600 \mathrm{~m}$, and slope angles of $15^{\circ}, 30^{\circ}, 45^{\circ}, 60^{\circ}$ and $75^{\circ}$. A total of 25 slope models are established, and the calculation main profile is shown in Figure 1. In order to reflect the dynamic response law of slope in displacement, velocity and acceleration amplification factors under specific conditions more simply and intuitively, the nodes at special parts of slope are monitored, as shown in Figure 1. And the dynamic response law of slope is reflected by comparing the displacement, velocity and acceleration amplification factors along the vertical direction (A1-A7) of slope top and along the slope direction (B1-A7).

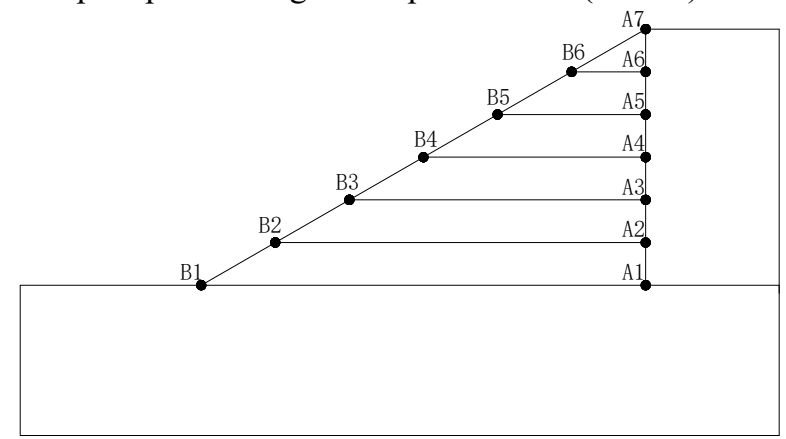

Figure 1. Slope model and monitor point

\section{Input of slope dynamic load and selection of calculation parameters}

In this paper, the acceleration time history used to simulate the dynamic response law of rock slope is 
shown in Formula (1). The propagation direction of cosine wave is vertically upward, and the particle motion direction is horizontal.

$$
a=0.5 g \cos (2 \pi f t)
$$

In the formula, $f$ is the seismic frequency, taking the value of $5 \mathrm{HZ}$. The amplitude is $0.5 \mathrm{~g}$. And the seismic wave duration takes the value of $10 \mathrm{~s}$.

It is assumed that the slope is a rock one with dynamic elastic modulus of $5 \times 10^{4} \mathrm{~Pa}$. Poisson's ratio is 0.2 and the density is $2500 \mathrm{~kg} / \mathrm{m} 3$.

The viscous boundary and the free field boundary are selected as boundary conditions. The viscous boundary is applied at the bottom of the model, and the free field boundary is applied around the rest of the model. Therefore, it is necessary to convert acceleration time integration into velocity time history as shown in Formula (2), and then into corresponding stress time history according to Formula (3).

$$
\begin{gathered}
v_{s}=-\frac{0.5 g}{2 \pi f} \sin (2 \pi f t) \\
\sigma_{s}=-2 \rho C_{s} v_{s}=\frac{g}{2 \pi f} \sqrt{\frac{E \rho}{2(1+v)}} \sin (2 \pi f t)
\end{gathered}
$$

\section{Study on dynamic characteristics of rock slope}

response

Symbols $\varepsilon, \xi$ and $\eta$ are selected to represent displacement amplification factor, velocity amplification factor and acceleration amplification factor respectively. Assuming that the maximum displacement value of any node $E$ in the slope during dynamic calculation is $D_{E}$ and the maximum displacement value of Node $C$ at the slope foot is $D c$, the displacement amplification factor of this node will be $\varepsilon=D_{E} / D c$. Similarly, the principle of the definitions of velocity amplification factor and acceleration amplification factor are the same as that of displacement amplification factor. Therefore, displacement, velocity and acceleration can be obtained by monitoring the displacement, velocity and acceleration values of each node in the dynamic calculation process, and then amplification factors of displacement, velocity and acceleration at each node can be obtained. The dynamic response law of slope can be reflected through the analysis of the three amplification factors.

\subsection{Influence of rock slope height on dynamic response characteristics}

In order to study the influence of slope height on seismic response of slope, five slope heights, $30 \mathrm{~m}, 60 \mathrm{~m}, 150 \mathrm{~m}$, $300 \mathrm{~m}$ and $600 \mathrm{~m}$, are taken respectively, and the slopes are all $30^{\circ}$.

It can be seen from Fig. 2 that when the slope height is $30 \mathrm{~m}$, the displacement amplification factor gradually increases up along the slope, and the displacement amplification amount increases from the slope foot to the slope shoulder 1.07 times; The distribution of velocity amplification factor has laws similar to displacement amplification factor, but the velocity amplification factor is slightly larger, reaching 1.16 times at the slope shoulder; The distribution of acceleration amplification factor has a similar law, too, and the acceleration factor increases more rapidly when it is near the slope shoulder, reaching 1.26 times.

When the slope height is $60 \mathrm{~m}$, the distribution forms of displacement amplification factor, velocity amplification factor and acceleration amplification factor are basically the same as those when the slope height is $30 \mathrm{~m}$, but the amplification value of the $60 \mathrm{~m}$-high slope is larger than $30 \mathrm{~m}$.

When the slope height is $150 \mathrm{~m}$, the distribution forms of displacement amplification factor, velocity amplification factor and acceleration amplification factor of slope dynamic response change obviously. Up along the slope, the displacement amplification factor gradually decreases at the beginning, and when it is about $40 \mathrm{~m}$ away from the slope foot, the displacement amplification factor is the smallest, and then gradually increases. When it reaches the slope shoulder, it reaches the maximum. The velocity amplification factor is almost constant along the slope direction.

When the distance from the foot of the slope is about $70 \mathrm{~m}$, the velocity amplification factor starts to increase gradually up along the slope, and reaches the maximum at the slope shoulder, which is close to 1.35 . The distribution law of acceleration amplification factor is exactly the same as that of velocity amplification factor.

When the slope height is $300 \mathrm{~m}$, a new situation appears in the dynamic response law of the slope. The displacement amplification factor gradually increases up along the slope. It increases to a maximum value of 1.05 in the range of $0 \sim 50 \mathrm{~m}$ from the slope foot. The displacement amplification factor gradually decreases to a minimum value of 0.83 in the range of $50 \sim 100 \mathrm{~m}$. And it increases gradually in the range of $100 \sim 300 \mathrm{~m}$ and reaches the maximum value of 1.5 at the slope shoulder. The velocity, acceleration amplification factor and displacement amplification factor have similar changes, which up along the slope, first increase, then decrease, reach a maximum and a minimum on the half way of the slope, and then increase again.

For a slope with a slope height of $600 \mathrm{~m}$, the distribution forms of displacement amplification factor, velocity amplification factor and acceleration amplification factor are basically the same as those when the slope height is $300 \mathrm{~m}$, that is, starting from the slope foot, up along the slope, reach some extremum on the slope, and start to enlarge near the slope shoulder, showing the rhythmicity of spatial intensity distribution of slope dynamic response. 


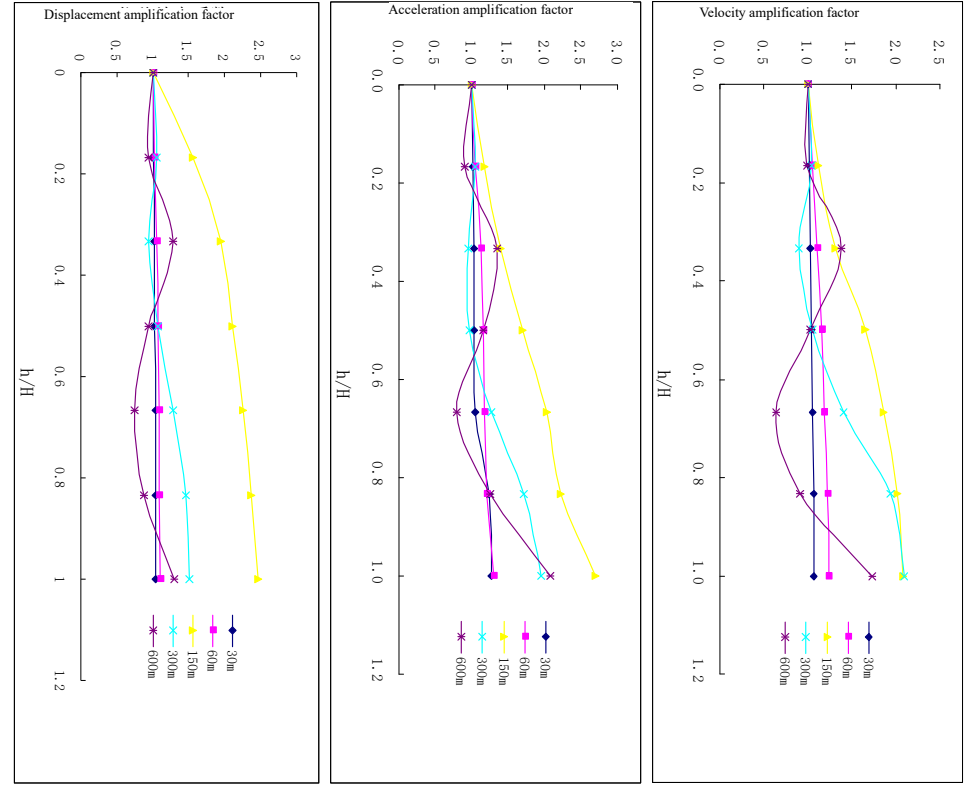

Figure 2. Relationship between the amplification factor of displacement, velocity, acceleration and between the slope height ratio under different slope heights

It can be seen from Figure 3 that the amplification factors of displacement, velocity and acceleration at the top of the slope do not increase with the increase of slope heights, but increase rapidly with the increase of slope heights in the range of the slope height $30 \sim 150 \mathrm{~m}$, decrease rapidly with the increase of slope heights in the range of $150 \sim 300 \mathrm{~m}$, and decrease with the increase of slope heights in the range of $300 \sim 600 \mathrm{~m}$, which, however, don't change much.
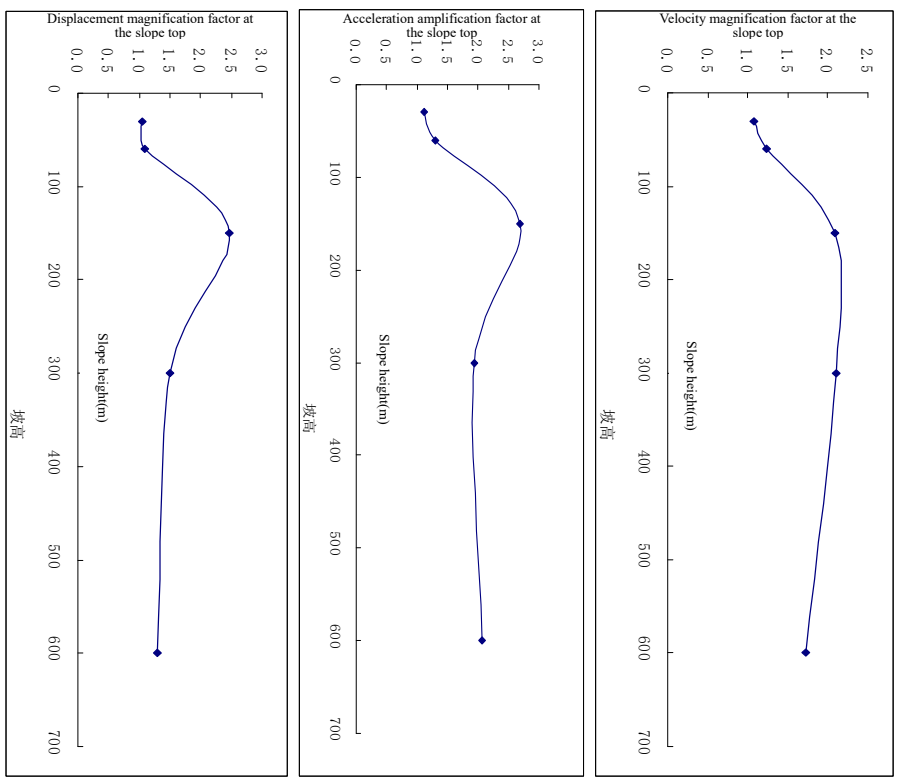

Figure 3. Relationship between the slope heights and the slope shoulder's amplification factors of displacement, velocity and acceleration

is $30 \mathrm{~m}$, the displacement amplification factor at the slope

\subsection{Influence of Rock Slope Gradient on Dynamic Response Characteristics}

In order to study the influence of slope gradient on the seismic response of slope under earthquake load, five slopes of $15^{\circ}, 30^{\circ}, \quad 45^{\circ}, \quad 60^{\circ}$ and $75^{\circ}$ are selected respectively for the five slope heights of $30 \mathrm{~m}, 60 \mathrm{~m}$, $150 \mathrm{~m}, 300 \mathrm{~m}$ and $600 \mathrm{~m}$ to monitor amplification factors of displacement, velocity and acceleration at the slope shoulders.

It can be seen from Fig. 4 that when the slope height shoulder does not change much with the increase of slope, which is about 1.07 times; the distribution of velocity amplification factor has laws similar to displacement amplification factor, but the velocity amplification factor is slightly larger, about 1.16 times; The acceleration amplification factor gradually increases with the increase of slope, and reaches the maximum value of 1.49 when the slope is $75^{\circ}$.

When the slope height is $60 \mathrm{~m}$, the displacement amplification factor does not change much with the increase of slope, about 1.17 times; The velocity amplification factor gradually increases to 1.45 times 
with the increase of slope between $15^{\circ}$ and $45^{\circ}$, and then tends to be stable. The acceleration amplification factor gradually increases with the increase of slope, and reaches the maximum value of 1.55 when the slope is $75^{\circ}$.

When the slope height is $150 \mathrm{~m}$, distribution forms of the displacement amplification factor and velocity amplification factor of the slope dynamic response do not change obviously. And the displacement amplification factor tends to be stable at the slope between $15^{\circ}$ to $45^{\circ}$, with an amplification factor of 1.16 times, and then increases slightly, but the increment is not significant. The velocity amplification factor increases with the increase of slope height, but the change is not obvious, ranging from 1.35 to 1.41 . The distribution form of acceleration amplification factor changes obviously, not linearly with the change of slope height. When the slope height is 45 , there comes out a minimum value of 1.40 , and then it rapidly increases to 1.84 .

When the slope height is $300 \mathrm{~m}$, the dynamic response law of the slope appears in a new situation with the change of slope. The displacement amplification factor, velocity amplification factor and acceleration amplification factor all have minimum values of 0.98 , 1.03 and 1.29 at the slope of $45^{\circ}$, and then increase rapidly with the increase of slope height, reaching the maximum values of $1.20,1.29$ and 1.69 at the slope of $75^{\circ}$.

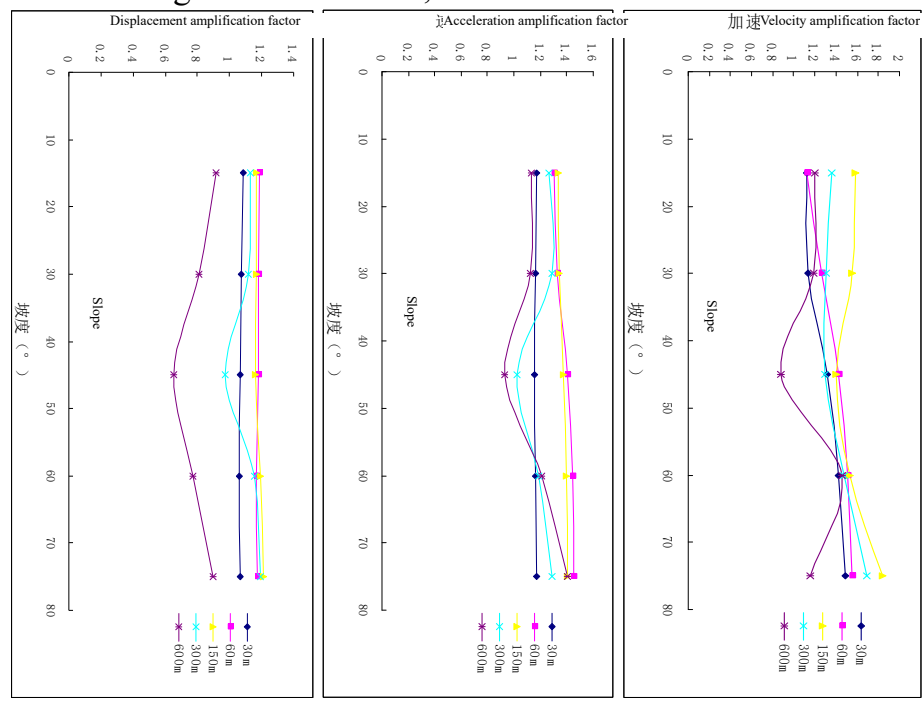

Figure 4. Relationship between the slope shoulder's amplification factor of displacement, velocity, acceleration and the slope angle under different slope heights

For a slope with a slope height of $600 \mathrm{~m}$, the distribution forms of displacement amplification factor and velocity amplification factor with slope height are basically the same as those with a slope height of $300 \mathrm{~m}$, with minimum values of 0.65 and 0.93 respectively, and maximum values of 0.90 and 1.41 respectively when the slope is $70^{\circ}$. There is a new distribution form of acceleration amplification factor coming out with the change of slope height. When the slope is between from $15^{\circ}$ to $45^{\circ}$, the amplification factor decreases with the increase of slope height, and a minimum value of 0.87 appears at the slope of $45^{\circ}$. When the slope is between from $45^{\circ}$ to $60^{\circ}$, the amplification factor increases with the increase of slope height, and a maximum value of 1.46 appears at the slope of $60^{\circ}$, and then decreases to the minimum value of 1.15 with the increase of slope height at he slope of $75^{\circ}$, which shows the rhythmicity of slope dynamic response distribution changing along with slope gradient.

\subsection{Influence of power input period on dynamic response characteristics}

Given the variation range of slope height is $30 \mathrm{~m} \sim 600 \mathrm{~m}$, the slope is $30^{\circ}$, and the variation range of power input period (reciprocal of frequency) is $0.2 \mathrm{~s} \sim 1 \mathrm{~s}$, investigate the distribution of dynamic response law of slope under the influence of different power input periods.

It can be seen from Fig. 5 that when the slope height is $30 \mathrm{~m}$, the displacement amplification factor decreases with the increase of the period length, ranging from 1.074 to 0.880 ; meanwhile, the distribution of velocity amplification factor has a law similar to displacement amplification factor, but the velocity amplification factor is slightly larger, ranging from 1.162 to 1.030; The acceleration amplification factor increases with the increase of the period length, which increases obviously from 1.139 to 1.364 in the range of the period length from $0.2 \mathrm{~s}$ to $0.4 \mathrm{~s}$, and then tends to be stable.

When the slope height is $60 \mathrm{~m}$, the variation law of displacement amplification factor and velocity amplification factor changing with the period length is similar to that of slope height of $30 \mathrm{~m}$, both of which decrease with the increase of the period length. The acceleration amplification factor decreases with the increase of the period length when the period length is in the range from $0.2 \mathrm{~s}$ to $0.6 \mathrm{~s}$, changing from 1.259 to 1.180 , and then starts to increase with the increase of the period length, and reaches the maximum value of 1.361 when the period length is $0.8 \mathrm{~s}$. 

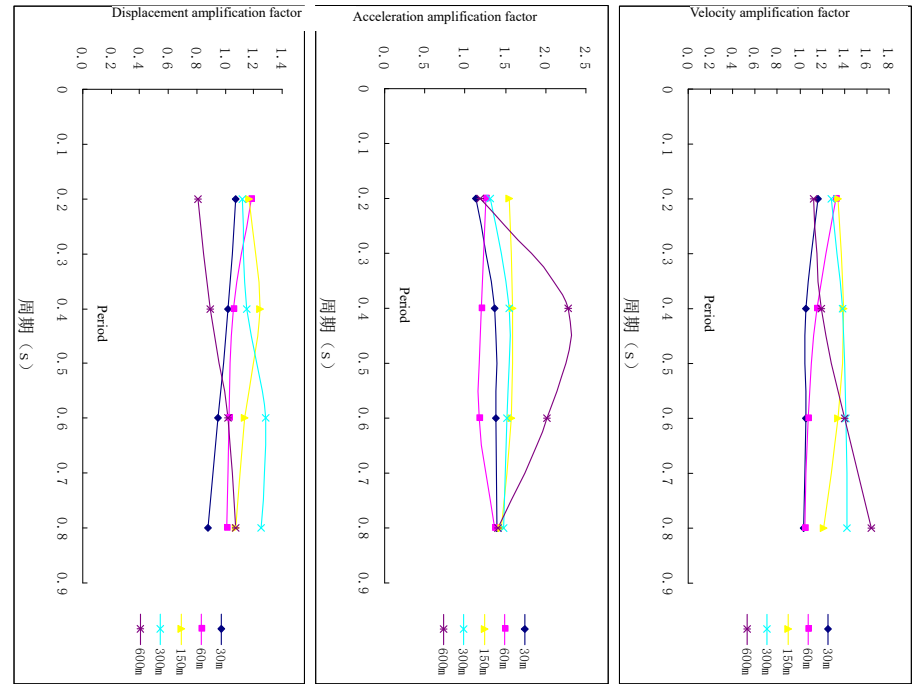

Figure 5. relationship between the slope shoulder's amplification factor of displacement, velocity, acceleration and the dynamic load period under different slope heights

with a slope of $30^{\circ}$ and a height is $600 \mathrm{~m}$ under the condition of acceleration time history $a=0.1 g \cos (2 \pi t / T)$

\subsection{Influence of dynamic input amplitude on} dynamic response characteristic

In order to discuss the influence of dynamic input amplitude on dynamic response of slope, two dynamic acceleration time histories, $a=0.1 g \cos (2 \pi t / T) \quad(T=0.2 \mathrm{~s})$ and $\mathrm{a}=0.5 \mathrm{~g} \cos (2 t / T) \quad(T=0.2 \mathrm{~s})$, are taken for studying respectively.

Fig. 6 shows the dynamic response law of the slope and the period of $0.2 \mathrm{~s}$. The former is the absolute value contour map of the three quantities (displacement, velocity and acceleration), the latter is the amplification factor contour map of three quantities, and Fig. 7 is the absolute value contour map and amplification factor contour map when the amplitude is $0.5 \mathrm{~g}$.

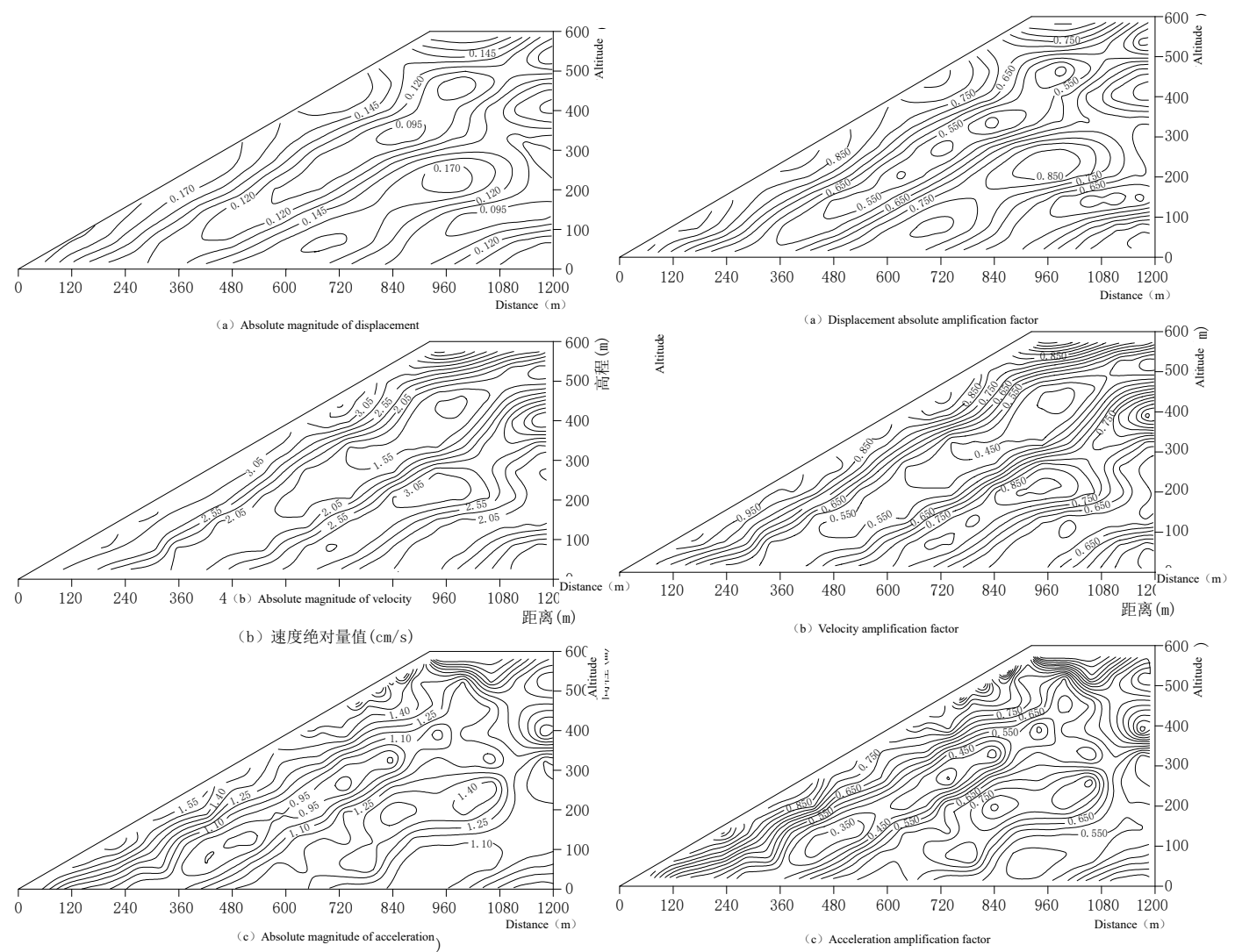

Figure 6. absolute magnitude and amplification factor contour map of the displacement, velocity, acceleration under the amplitude of $0.1 \mathrm{~g}$ 

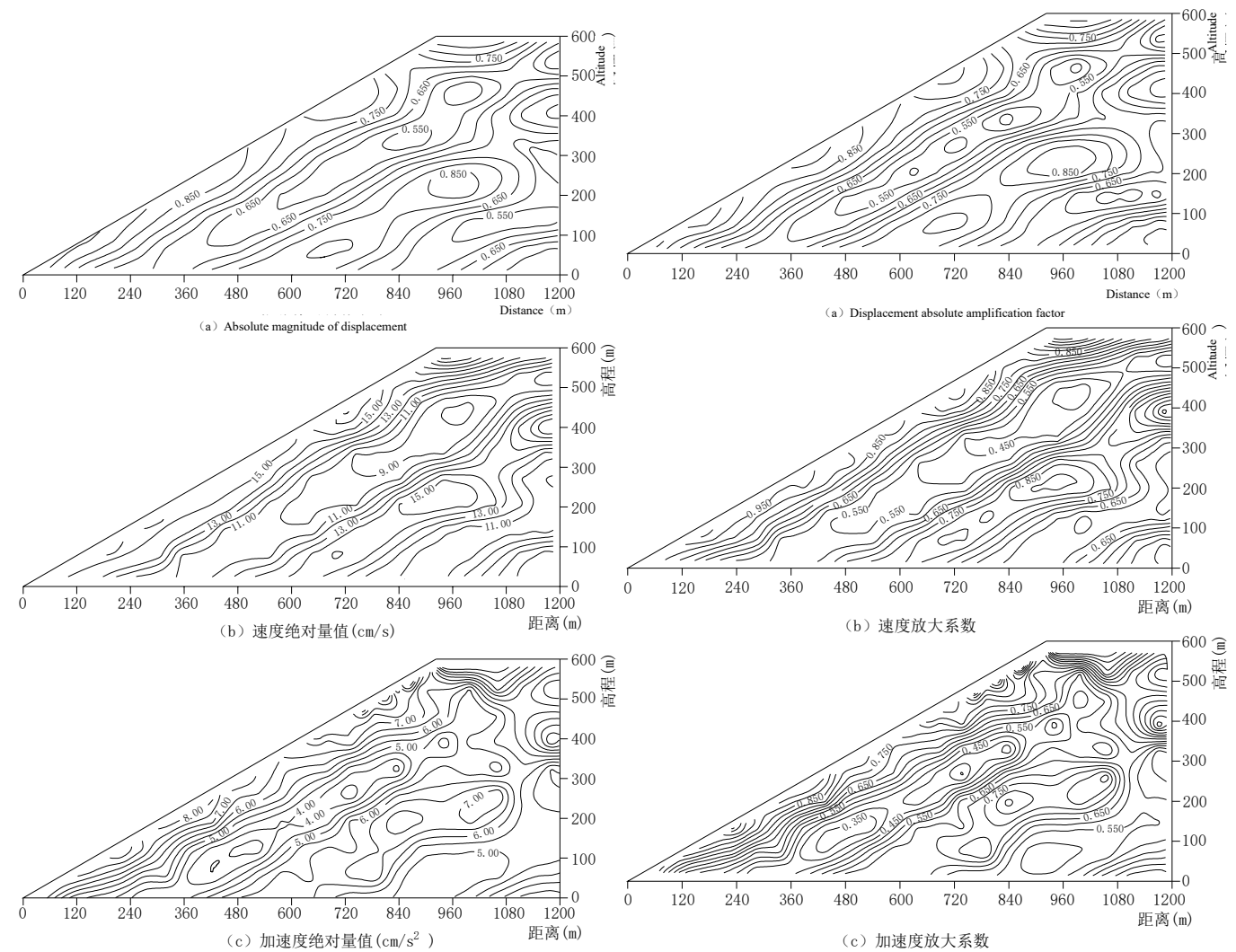

Figure 7. absolute magnitude and amplification factor contour map of the displacement, velocity, acceleration under the amplitude of

By comparison, it is not difficult to find that the absolute magnitude of the quantities (displacement, velocity and acceleration) in Fig. 7 are 5 times of those of corresponding particles in Fig. 6, while the amplification factors of displacement, velocity and acceleration in Fig. 6 are basically the same as those in Fig. 7. This shows that the amplitude of seismic wave increases in direct proportion to the absolute values of displacement, velocity and acceleration, but does not affect the distribution of amplification factors of displacement, velocity and acceleration of each node on the slope.

\section{Conclusion}

In this paper, FLAC ${ }^{3 D}$ is used to study the dynamic response law of slope, considering the influence of different slope heights, slope, seismic wave period and seismic wave amplitude on the dynamic response law of slope, and the following conclusions have been drawn:

(1) Different heights. When the slope is low $(30 \mathrm{~m}$, $60 \mathrm{~m}, 150 \mathrm{~m}$ ), displacement, velocity and acceleration increase with the increase of slope height up along the vertical direction. However, when the slope is higher $(300 \mathrm{~m}, 600 \mathrm{~m})$, the amplification factors of the three quantities (displacement, velocity and acceleration) are not linearly amplified up along the elevation in the vertical direction, but sometimes increase, sometimes decrease, increasing and decreasing alternately, forming some Extremum closed cycles. Moreover, the maxima and minima appear alternately, showing rhythmic changes in spatial ranges.

(2) Different slopes. The dynamic response law of
$0.5 \mathrm{~g}$

slope is different when changing slope angle under different slope heights. When the slope is low $(30 \mathrm{~m}, 60 \mathrm{~m}$, $150 \mathrm{~m}$ ), the change of slope has little influence on the amplification factors of the three quantities (displacement, velocity and acceleration) at the slope shoulder, while for higher slopes $(300 \mathrm{~m}, 600 \mathrm{~m})$, the amplification factors of displacement, velocity and acceleration are sensitive to the change of slope. Moreover, it does not increase or decrease linearly with the slope, and the acceleration amplification factor is more sensitive to the change of slope.

(3) Seismic wave period. Under different slope heights, the dynamic response law of slope is different when the period of seismic wave is changed. When the slope is low $(30 \mathrm{~m}, 60 \mathrm{~m}, 150 \mathrm{~m})$, the longer the period is, the slower the vibration frequency of wave is, and the amplification factor of displacement, velocity and acceleration at the slope shoulder generally show a decreasing trend. However, for higher slopes $(300 \mathrm{~m}$, $600 \mathrm{~m}$ ), the amplification factors of displacement, velocity and acceleration have no obvious law along with the periodic change, instead of linearly increasing or decreasing, there is a certain rhythm phenomenon.

(4) Seismic wave amplitude. Under the influence of the given factors such as slope height, slope angle and period, the amplitude of seismic wave increases in direct proportion to the absolute values of displacement, velocity and acceleration, but it does not affect the distribution of amplification factors of displacement, velocity and acceleration at each node on the slope. 


\section{Acknowledgement}

Fund Project: Tianjin Science and Technology Major Project(18ZXAQSF00110); National Natural Science Foundation (41772123)

\section{About the Author}

$\mathrm{Fu}$ Zhanling (born in December 1986), male, Han Nationality, born in Tianjin, serve as an engineer, Master Degree Candidate, has been engaged in the research on seismic exploration and earthquake engineering from perspectives of geophysics and geochemistry. E-mail: 375165658@126.com; Tel.: 13516179883. Address: Tianjin Earthquake Administration, No.19, Youyi Road, Tianjin. Postal Code: 300201.

\section{References}

1. Xu Guangxing, Yao Lingkan, Gao Zhaoning, et al. Large-scale shaking table model test study on slope dynamic characteristics and dynamic response[J].Journal of Rock and Soil Mechanics and Engineering,vol 3,2008.

2. Xiao Ruihua, Xu Qiang, Feng Wenkai, et al. Shaking table physical simulation test study on deformation and failure mechanism of double-sided slope under strong earthquake conditions[J].Journal of Engineering Geology,vol 6,2010.

3. Zhang Ping, Wu Delun.Experimental study on slope sliding under dynamic load[J].Journal of Chongqing Jianzhu University,vol 2,1997.

4. Ding Yanhui.Study on prediction method of earthquake landslide in western China[D].Beijing:China University of Geosciences, 1997.

5. Wang Cunyu,Wang Sijing.Study on the bank slope stability of Ertan Reservoir under earthquake conditions, problems of engineering geomechanics of rock mass (7)[M]. 1987, Beijing: Science Press.

6. He Yunlong, Lu Shuyuan. Approximate calculation method for seismic action of rock slopes[J]. Chinese Journal of Geotechnical Engineering, vol 2,1998.

7. Qian Shengguo, Lu Qiurong. Seismic stability analysis of the high slope of the Three Gorges Ship Lock on the Yangtze River[R]. Wuhan: Scientific Research Report of Wuhan Yangtze River Scientific Research Institute, 1991.

8. Li Yushu, Gao Guangyun, Li Tianbin. Seismic dynamic response and stability analysis of the slope of tunnel entrance under bias pressure[J].Journal of Underground Space and Engineering, vol 5,2006. 